\title{
PATHWAYS TO IMPROVED AERODYNAMIC DESIGN
}

\author{
James H. Page ${ }^{1}$, Paul Hield ${ }^{2}$, Callum Mantell ${ }^{1}$ and Paul G. Tucker ${ }^{1}$ \\ ${ }^{1}$ University of Cambridge \\ Department of Engineering \\ Trumpington Street \\ Cambridge, CB2 1PZ \\ United Kingdom \\ e-mail: $\{$ jhp41,crm61,pgt23\}@cam.ac.uk \\ ${ }^{2}$ Fan Systems Engineering \\ Rolls-Royce plc \\ Filton, Bristol, BS34 7QE \\ United Kingdom \\ e-mail: Paul.Hield@Rolls-Royce.com
}

Keywords: Graphics Processing Unit, Computational Fluid Dynamics, Turbomachinery, Distortion, Design.

\begin{abstract}
Large-scale unsteady graphical processor unit (GPU) based calculations are used to study the flow in realistic transonic fan geometries and shown to produce useful insights into the complex unsteady flow physics in systems of practical engineering relevance. Inlet flow distortion produces complex flow patterns which can significantly reduce stall margin. In order to design efficient, well targeted fan stabilizing technologies, it is essential to gain understanding into which specific features of distorted flow patterns are important in causing instability.

In this paper, the use of large-scale, high-fidelity 3D unsteady Reynolds-Averaged Navier Stokes (URANS) calculations with sliding planes is shown to allow the isolation of a wide range of inlet distortion features and a high throughput of calculations is achieved. This facilitates a comprehensive investigation into stall margin loss and the observation of complex instability processes, which is in turn used to support the development of specialized design solutions. The costs of simulations and turnaround times are given. The potential for mixed fidelity simulations, in particular mixing one-dimensional low order models and large eddy type simulations to generate inlet conditions, is discussed.
\end{abstract}




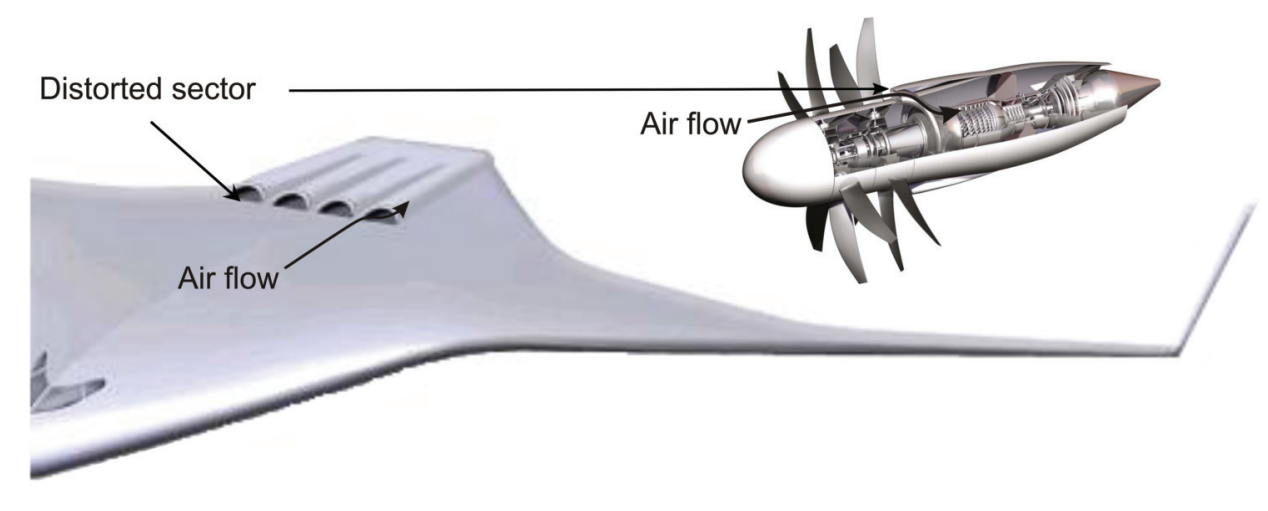

Figure 1: Aerospace airframe and propulsion technology under development.

\section{INTRODUCTION}

The world aircraft fleet is predicted to double by 2030.[1] At the same time, mankind faces the immense challenge of reducing $\mathrm{CO}_{2}$ and other emissions, along with other pressing tasks such as reducing noise.[1] The Advisory Council for Aeronautics Research in Europe (ACARE) has set targets to reduce $\mathrm{CO}_{2}$ and noise emissions $50 \%$ by 2020.[1] These challenges have proved to be a game changer in encouraging the development of promising alternative technologies and new concepts in aircraft design. Examples, among others, are the open rotor gas turbine engine and the boundary layer ingestion (BLI) Silent Aircraft, shown in figure 1. The emergence of such novel architecture raises a considerable deficit in aerodynamic understanding and extensive design spaces to explore. While experimental rigs lack flexibility and scope before such a challenge, high fidelity computational fluid dynamics (CFD) has the agility to aggressively explore and narrow down the vast design spaces.

CFD is a rapidly developing tool used to support aerodynamic component design in the aerospace industry. It is, however, not an exact science and its successful use requires prudent consideration of numerous sources of error, listed by Denton [2] as numerical errors due to finite difference approximations, modelling errors for example turbulence modelling, unknown boundary conditions, unknown geometry such as tip clearances or leading edge shapes, and assumptions of steady flow. Improvements in the capability of high performance computing and advances in CFD codes have made practical the deployment of unsteady 3D CFD on an industrial scale.

3D unsteady CFD comprises, in order of increasing fidelity, unsteady Reynolds-Averaged Navier-Stokes (URANS) calculation, detached eddy simulation (DES), large eddy simulation (LES) and Direct Numerical Simulation (DNS). While computational expense confines the last three in the list to academic study and very small scale industrial use, URANS has now become practical for large-scale studies in industrial aerodynamic design processes.

An important topic in jet engine design for futuristic aircraft architecture is inlet distortion due to curved intake ducts, which has the potential to reduce fan stall margin and negatively affect fan stability. There is a press need to gain in-depth understanding of distorted intake flow in order to design efficient, well targeted fan stabilizing technology. 

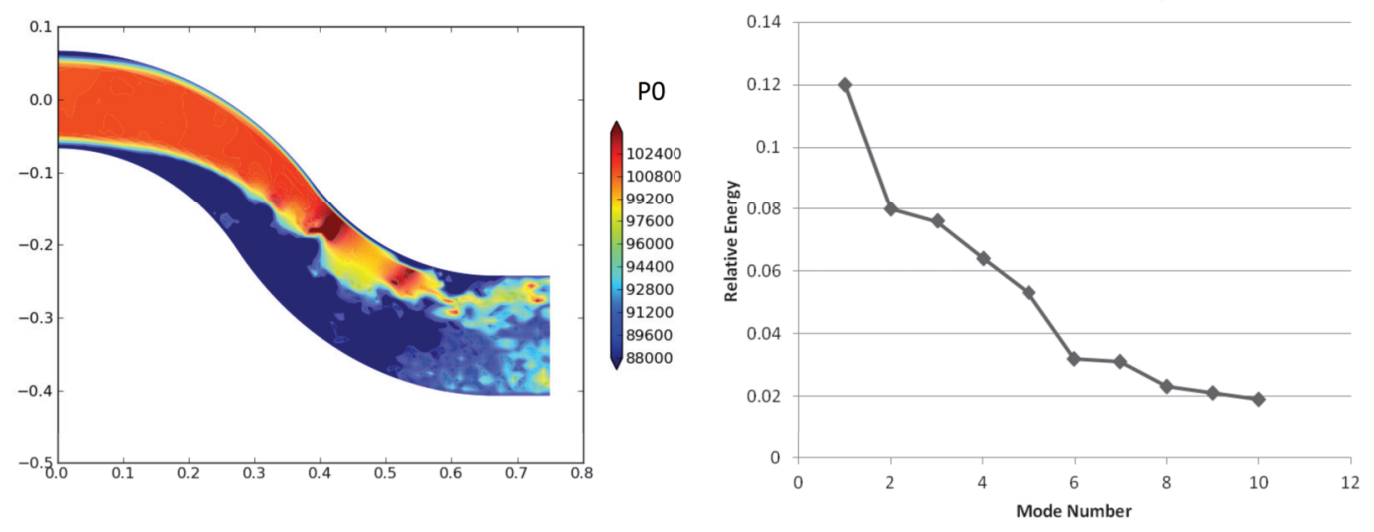

Figure 2: Azimuthal cut showing a typical curved duct LES flow.

\subsection{Inlet Distortion}

Research into the effects of an inlet total pressure circumferential square wave on stall margin was carried out with and without VIGVs by Shaw et al. [3]. They found that the distortion significantly reduced stall margin and that some of the reduction could be recovered by using VIGVs to reduce the circumferential variation in inlet absolute swirl. Other literature applicable to fan inlet distortion also looked at square waves. Mistry et al. [4] carried out experiments on a contra-rotating rotor rig and analysed effects on aerodynamics and performance. Gunn et al. [5] conducted experiments on a low speed fan stage and studied the effect on loss mechanisms. Gunn et al. [6] later studied the effect of BLI inlet profile on fan stage aerodynamics and loss using CFD and experiments. More recently, Perovic et al. [7] investigated stall inception in the same BLI environment, gaining insight in to the growth and decay of stall cells. So far, research has focussed on more or less idealized distortions while leaving out much of the detail in real flows.

What is now well known is that fan flow and intake flow are strongly coupled. Future design of curved intakes and fans will become increasingly integrated to ensure good performance in non-uniform unsteady intake flow. A large eddy simulation (LES) of flow in an S shaped curved duct based on that used by Garnier reveals a highly complex, unsteady distortion pattern emerging downstream of the curves.[8] The distortion is characterised by a static pressure and velocity distortion, 3D secondary flow vortices, total pressure losses in the boundary layer and main flow — both due to separation — and unsteady vortex shedding. These are visible in the azimuthal snapshot of total pressure $(P 0)$ in figure $2 .{ }^{1}$

A key question with respect to fan rotor stabilization is which particular distortion features are most damaging and what are the stalling mechanisms that they promote? To answer, it may be helpful to deconstruct inlet distortion and test each feature separately. A popular method for analyzing complex flows is proper orthogonal decomposition (POD) which deconstructs the cross-sectional unsteady velocity field into modes and quantify their relative energies. [8] Where one or a few modes dominate, this can be a powerful method of identifying dominant axially aligned vortical flow structures. However, the POD relative energy plot in figure 2 shows a broad spectrum with the most energetic 10 modes accounting for only half of the total energy. Therefore as an alternative, visual inspection of the flow suggests the following decomposition:

\footnotetext{
${ }^{1}$ Results kindly provided by R. Watson.
} 
1. inlet vortices of various sizes and orientations,

2. the static pressure and associated velocity distortion due to curved streamlines,

3. a total pressure deficit due to separation in the curved intake in addition to the above static pressure and velocity distortion,

4. a time-averaged distortion profile from the Garnier duct LES, which includes more realistic total pressure 2D spatial and temporal profile due to separation in addition to the curved streamline static pressure and velocity distortion,

5. the unsteady Garnier duct LES profile which adds a complex unsteady dimension to the time-averaged version.

Further research on complex distorted flows and methods of analysis is underway.

Building on item 5, a way to couple the URANS fan flow to the LES duct flow is to input the duct outflow directly into the fan inlet. Results for this will be shown in section 3.4. However, a far better way would be to replicate the particular fan rotor in the duct LES downstream conditions by means of an inexpensive body force model such as that proposed by Cao et al. [9]. This would allow the fan to impose its own influential static pressure field on the duct flow which would generate more realistic inlet conditions for the fan URANS calculation.

A full account of the analyses of each part is beyond the scope of this paper. Therefore, calculations for items 2, 3 and 4 will be discussed in section 3 .

\subsection{The Use of URANS in Aerodynamic Design and Fan Stall}

An extensive validation of the URANS solver with consistent settings and the Spalart-Allmaras turbulence model was carried out under the same project umbrella on a Rolls-Royce single stage fan rig by Shaw et al. [3]. The validation fan geometry was similar in pressure ratio and aspect ratio to the geometry used in this paper. The CFD was found to show good agreement with experimental results in radial and circumferential total temperature and total pressure profiles, and changes in stall margin. This is regarded to provide sufficient validation for the further project work in this paper.

In an internally reported work, further studies were carried out using two different CFD solvers each with the Spalart-Allmaras and mixing length turbulence model. Clean stall was predicted to within $9 \%$ of the test nozzle area in $95 \%$ of about 40 different fan geometries. Most importantly, however, the results for change in stall margin under the influence of inlet distortion were within $3 \%$ of the experimental results.[3]

The key point is that, while predicting single values on a large scale is still out of reach, URANS is sufficiently powerful method to investigate behaviour patterns of the level of complexity of stall. This will be shown to be useful in supporting design choices and providing significant understanding of the design space prior to experimental investigation.

\section{METHODOLOGY}

\subsection{Fan Geometry}

A series of CFD experiments have been carried out on the rotor of an advanced two-stage transonic fan geometry, which was inverse designed by the methodology and code described in Page et al. [10]. The geometry, shown in figure 3, was designed to specified radial profiles for stage pressure ratio and exit flow angle, and a specified mass flow at a nominal $100 \%$ speed. 

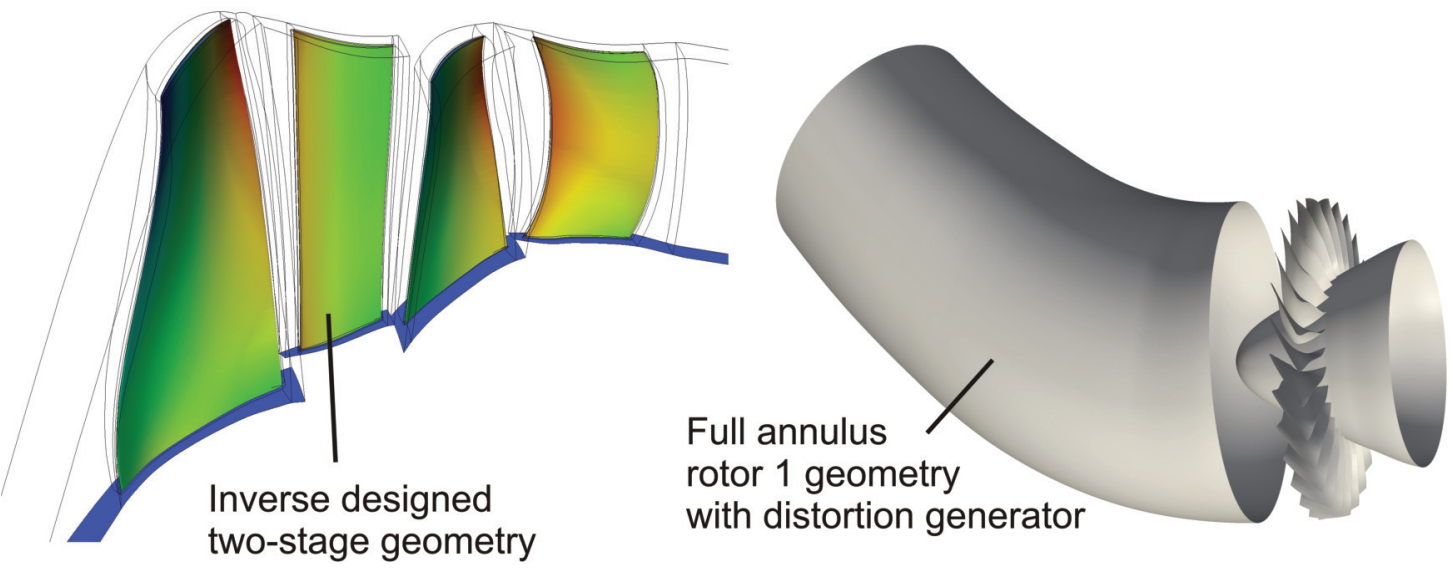

Figure 3: The two-stage fan geometry designed by a dual point inverse design algorithm and the 3D URANS rotor geometry with distortion generator.

Using the dual point algorithm developed by the authors, the fan was designed to simultaneously achieve these performance targets at $100 \%$ speed and desired rotor shock positioning, blade loading distribution and, therefore, peak efficiency at $90 \%$ speed. The design to mass flow and radial stage pressure ratio and exit flow angle profiles for this geometry were chosen to be representative of typical Rolls-Royce fans. The design is described in greater detail in [10].

For the CFD calculations in this paper, the first rotor blade was converted to a multipassage geometry. Figure 3 also visualizes the full annulus rotor geometry.

\subsection{Inlet Distortion Generator}

The method used to generate distortion items 2 and 3 is a curved intake duct attached upstream of the full annulus rotor as shown in figure 3 . The duct is area ruled with the cross-section transitioning from elliptical to circular and features a bullet formed by a second order Bezier surface of revolution. The walls can be defined as either inviscid slip walls or viscous no-slip. Part way along the duct an impermeable fence can be positioned in the flow if so desired.

The purpose of the inviscid slip walls was to facilitate the generation of the static pressure and velocity distortion associated with curved streamlines without the addition of total pressure distortion, especially a thicker boundary layer on the inside of the curve. With the no-slip walls a circumferentially non-uniform boundary layer was permitted.

\subsection{Stall Point Calculation}

The CFD calculations in this paper were carried out unsteady using Turbostream, a URANS CFD solver.[11] The code uses the finite volume method to solve for the flow on structured O-H multiblock meshes with hexahedral cells. The temporal discretization used in unsteady mode is based on the second-order Adams-Bashforth scheme. For this paper, all the calculations were carried out unsteady with a CFL number of 0.3 and using the Spalart-Allmaras turbulence model. Wall functions were used at solid boundaries. Sliding planes were used to interface rotating rotor mesh blocks with static mesh blocks for static blade rows or intake ducts.

In order to calculate the stall point, a choked nozzle was used to control the exit flow function and thus the position on the characteristic. Starting from a nozzle area away from the stall point, unsteady CFD was calculated over six revolutions, representing about three through-flows, with 


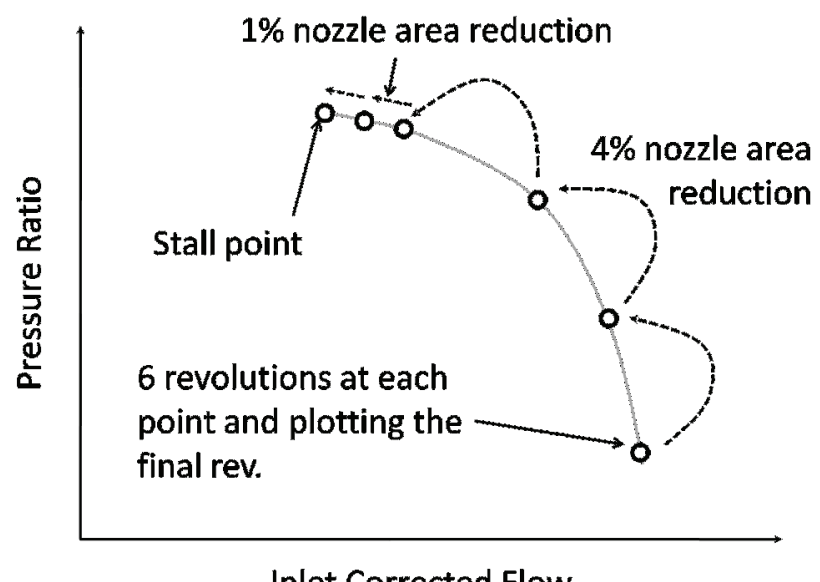

Inlet Corrected Flow

Figure 4: METHOD USED TO PLOT A CHARACTERISTIC CURVE AND CALCULATE THE STALL POINT.

flow results being saved once every blade passing during the final rev. The nozzle area was then reduced by $4 \%$ and the six rev procedure repeated. The stall point was calculated by successive $4 \%$ nozzle area reductions in this manner, followed by a targeted $1 \%$ nozzle area reduction high resolution pass. The procedure is illustrated in figure 4

The pressure ratio versus corrected inlet mass flow for each blade passing provided a cloud of points for each nozzle area showing the point on the characteristic curve along with the level of unsteadiness.

\subsection{High Performance Computing Architecture}

Turbostream is an updated version of TBLOCK, recoded and optimized for Graphics Processing Units (GPUs), delivering a speedup of typically up to 20 times.[11] The hardware used to carry out the calculations was a cluster comprising 48 Nvidia K10 GPUs. Each K10 is made up of two GK104 GPUs, each of which has 1536 cores. The investigation required the calculation of six nozzle areas on a one-third annulus geometry and 24 nozzle areas on a full annulus geometry. In total there were the equivalent of 156 full annulus revolutions.

\section{THE EFFECT OF INLET DISTORTION FEATURES ON ROTOR STALL MARGIN}

\subsection{Clean Inlet Stalling Behaviour}

The first CFD experiment calculated the stall point of the 'clean inlet' case. As the clean inlet was axisymmetric, the unsteady calculation was run on one-third of the annulus. The stall inception was observed to be due to tip leakage flow disturbing the flow at the leading edge of the blade[12] near the tip, leading to separation and the formation of a rotating stall vortex[13] which develops into a rotating stall cell. Figure 5 uses the Q-criterion vortical structure visualization to show separation close to the tip and the rotating stall vortex which is formed moments afterwards. Figure 6 shows the velocity field superimposed on axial velocity contours, highlighting the strong negative axial component of velocity (blue) interfering with the inlet flow in the tip region near the leading edges. 


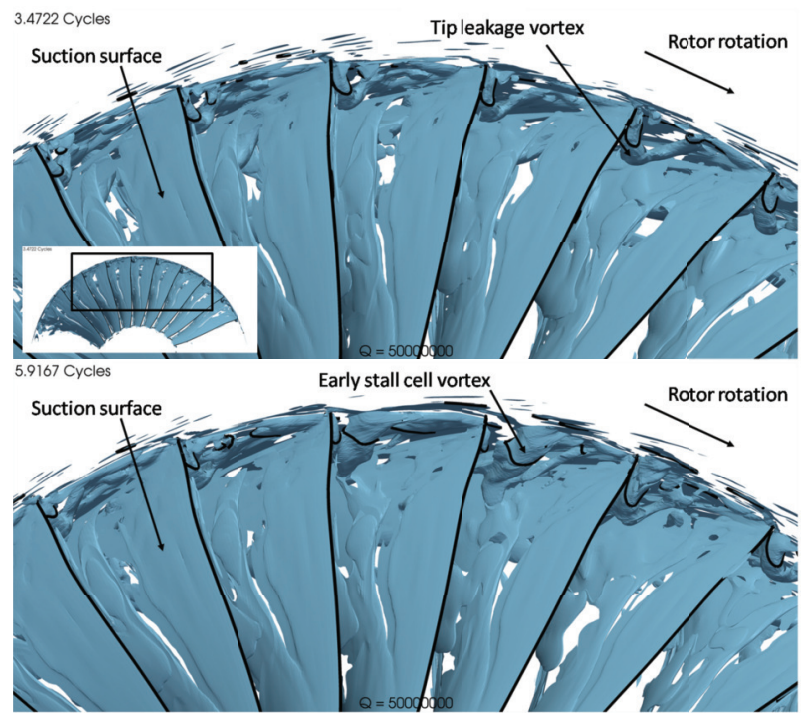

Figure 5: Vortical structure visualization using Q-criterion at stall inception.

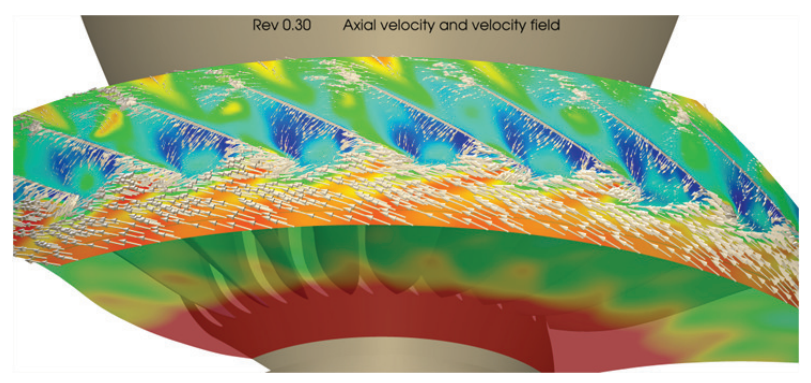

Figure 6: Tip region velocity field and axial velocity contours.

\subsection{Curved Streamline Static Pressure and Velocity Distortion}

The second CFD experiment compares the stall point of the inviscid slip-wall distortion generator case with the clean inlet case. The azimuthal plot in figure 7 shows the distortion generated by the curved duct. The variation between the higher static pressure and lower axial velocity on the outside of the bend and the lower static pressure and higher axial velocity on the inside of the bend is clearly visible. It is also noted that there is the absence of an inlet boundary layer or separation and the total pressure profile remains largely uniform. The curved duct isolates the static pressure-velocity distortion feature. The axial velocity seen by the rotor is shown again in the axial cut in figure 7 . The upward trajectory of the incoming flow leads to a swirl distribution with regions of co-swirl and contra-swirl entering the rotor. This is evident in the tangential velocity plot in figure 7 which shows a negative swirl velocity, co-rotating with the rotor, on the left and a positive swirl velocity, contra-rotating against the rotor, on the right. The absence of a boundary layer is again conspicuous.

Figure 7 shows a remarkably well behaved flow at $95 \%$ span via relative Mach at the last stable point on the characteristic. This case required six revolutions to be calculated at each of six nozzle areas. The wall clock time per revolution averaged 3 hours 11 minutes on 24 K10 GPUs, giving 19 hours 6 minutes per nozzle area and 114 hours 36 minutes (less than 5 days on half of the cluster) to calculate the stall point. This case showed no significant change in stall 

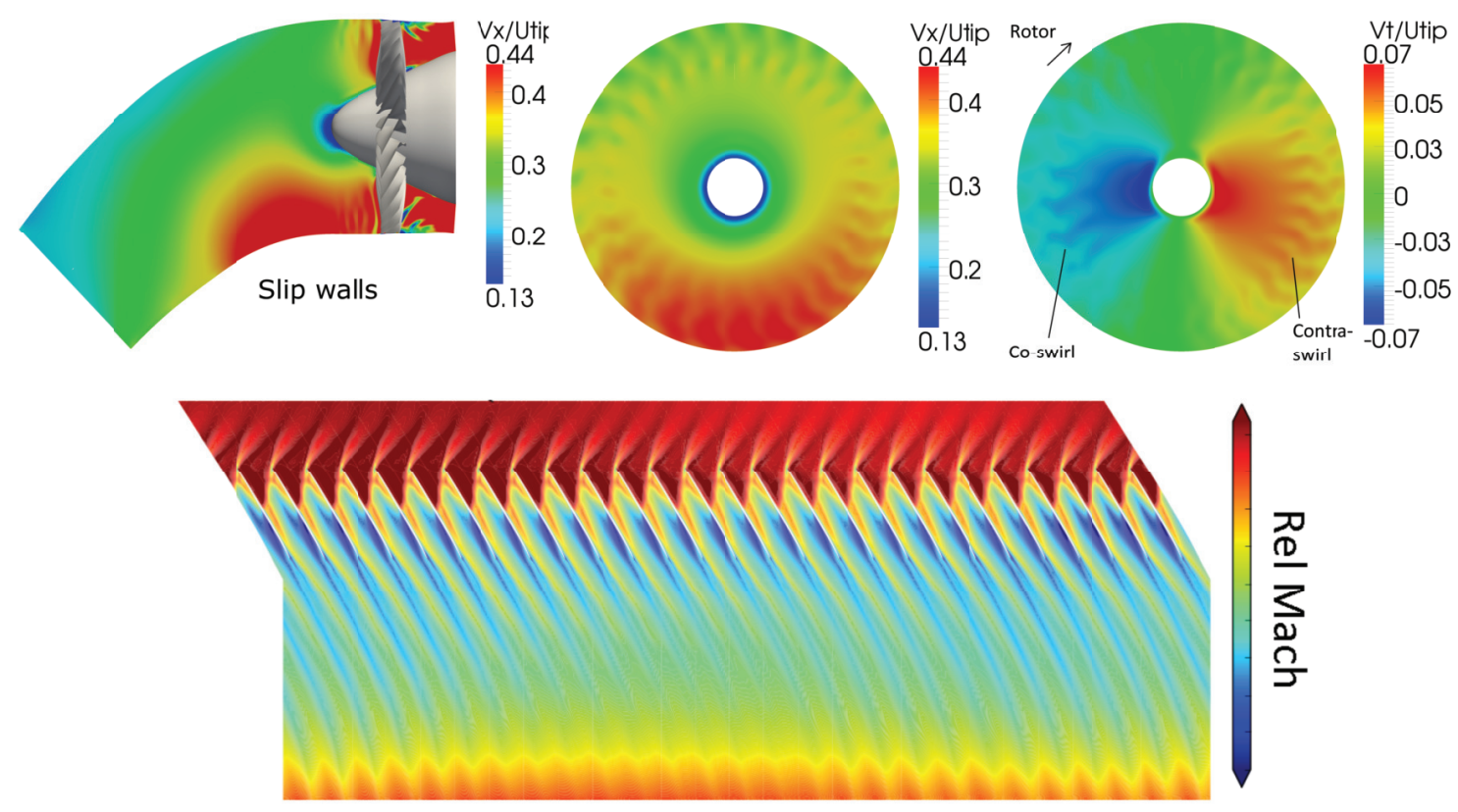

Figure 7: Overview of the inlet and fan flow with curved streamline distortion.

margin from the clean inlet, ruling out the importance of distortion due to curved streamlines alone at this magnitude.

\subsection{Curved Streamline Static Pressure and Velocity Distortion with Boundary Layer}

The third CFD study assessed the effect of adding a circumferentially varying boundary layer to the curved streamline distortion pattern. No-slip walls were added to the curved distortion generator to modify the inlet profile to include the thin boundary layer which is visible on the inside of the curve in figure 8. The circumferential profile of the new distortion and the swirl is shown in figure 8 to be very similar to the previous case but with the boundary layer visible. A key feature of the inlet profile is that is now contains total pressure loss near the tip, which is most pronounced downstream of the inside of the curve.

The radial plot of relative Mach at the final stable nozzle area shows how much more separated the flow is compared with the previous case. The turnaround time for this case was only fractionally longer than for the previous case. The stalling point for this distortion was at a nozzle area $3 \%$ larger than with clean, giving a key result in identifying low momentum tip flow as a important distortion feature. 

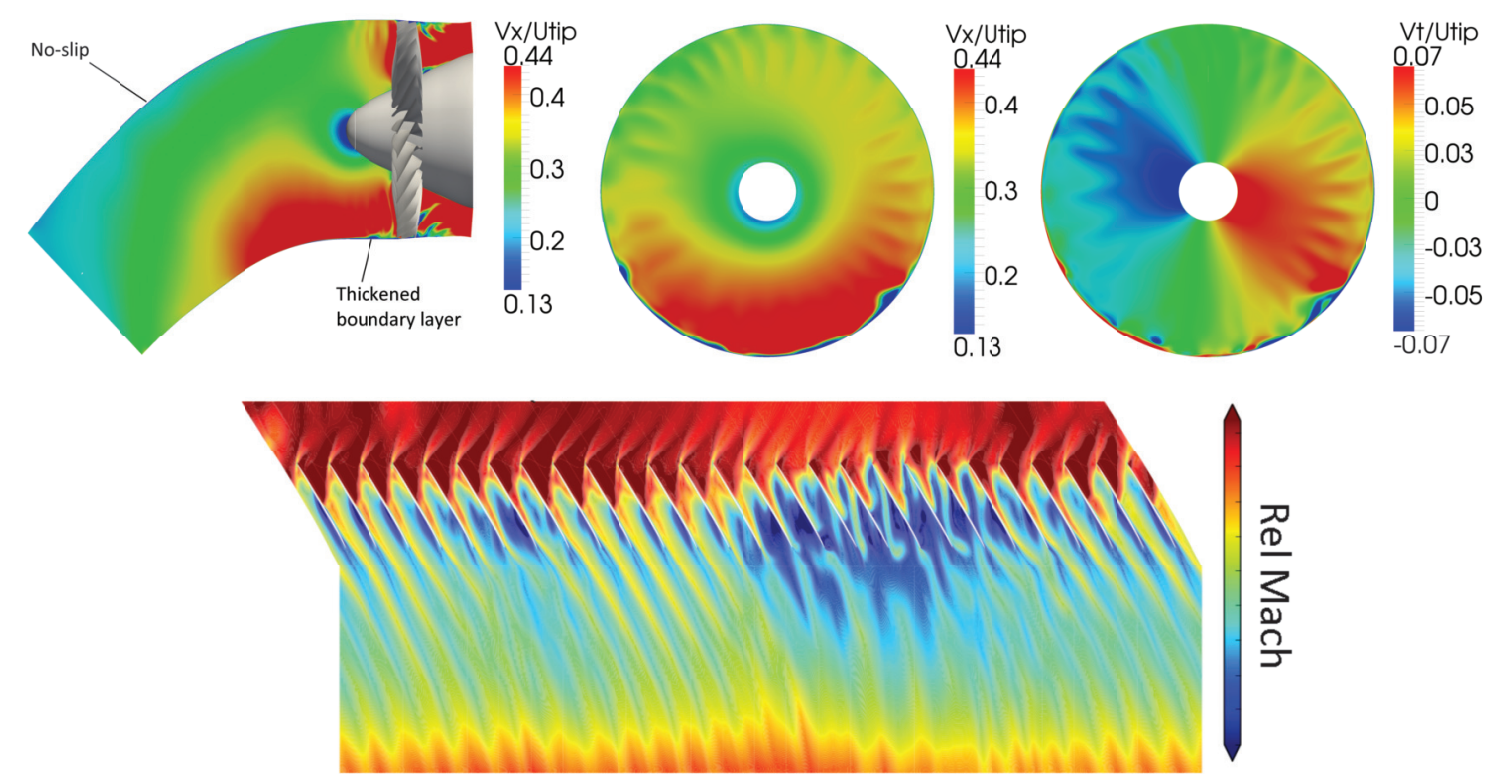

Figure 8: Overview of the inlet and fan flow with curved streamline distortion and boundary layer.

\subsection{Garnier outlet flow as fan inlet condition}

The final increment in the increasing complexity of inlet conditions shown in this paper is where the Garnier LES outflow is input directly into the rotor inlet. Six revolutions of unsteady Garnier duct flow data were input to straight duct section upstream of the fan rotor. Figure 9 shows snapshots of the inlet and fan flow, clearly demonstrating its chaotic nature.

The turnaround wall clock time for calculations increased to an average of 5 hours 35 minutes per revolution on $24 \mathrm{~K} 10$ GPUs, giving 33 hours 30 minutes per nozzle area or 134 hours to find the stall point with four nozzle areas. The increase in time per revolution was due to the highly unsteady flow field requiring a greater average number of inner time-steps to converge. In this case each point in the characteristic displayed increased unsteadiness but there was no significant reduction in stall margin compared with the curved duct with boundary layer. The Garnier duct flow is essentially a combination of distortion features including low momentum tip region flow. The lack of a further drop in stall margin in the presence of this inlet flow provided an early suggestion that the low momentum flow in the tip region encountered in the previous case was could still by far the most important flow feature. 

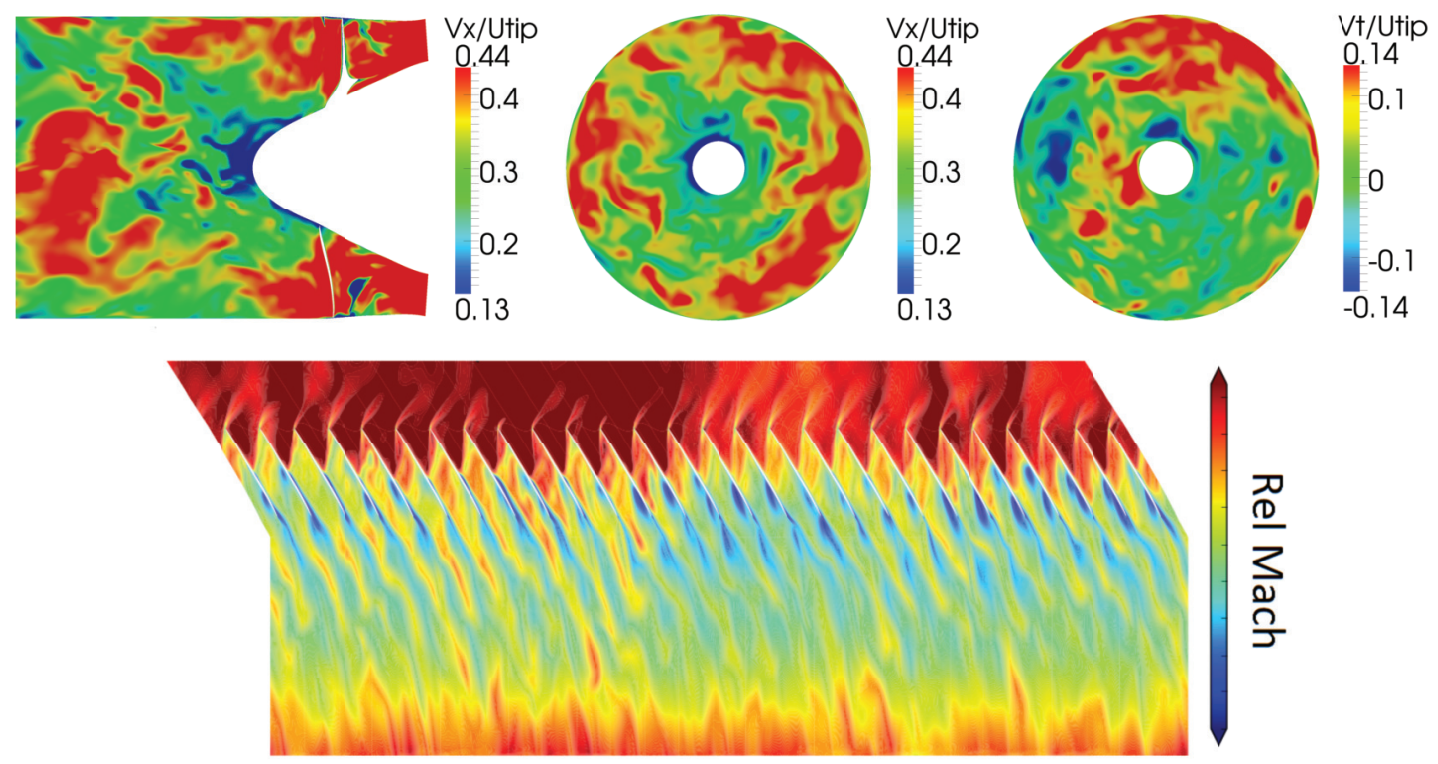

Figure 9: Overview of the inlet and fan flow with a straight inlet the Garnier results as inlet conditions.

\section{Conclusions and Discussion}

This paper discusses developments in the use of CFD in gas turbine engine design and demonstrates the practicality and value of large-scale 3D URANS in the design process via a non-exhaustive outline of an investigation into fan stall with inlet distortion. Results from LES of the Garnier duct were used to support the design of idealized distorted inlet conditions and to provide a complex unsteady inlet profile. This highlighted low momentum tip region flow as a key distortion pattern.

Resulting from this work, a recirculation passage geometry similar to that described by Guinet et al. [14, 15] has been designed and simulated. Design features of the passage are currently under URANS investigation by the authors and are providing early evidence of a significant increase in stall margin. Figure 10 shows a the plan view of the recirculation passages on a 3D multipassage geometry. The passage was preliminarily designed using $1 \mathrm{D}$ diffuser theory [16] to diffuse the flow and reduce losses in the passage, before reaccelerating to maintain attachment around a low radius curve and achieve high velocity flow injection. The diffuser sections are marked in the side view of the passage in figure 11. The diffuser sections 3D URANS in a one-third annulus geometry was then used to calculate stall points for variations in passage width, injection yaw angle and circumferential positional distribution.

One improvement in the current design process should be the calculation of more realistic inlet conditions for the fan. A duct flow LES using a body force representation of the fan rotor, as mentioned in section 1.1, would create far more realistic outlet conditions than a static pressure condition by coupling duct and rotor flows. Figure 12 compares schematic diagrams of the current and future setups.

A second improvement would be to increase the fidelity of the CFD local to stalling mechanisms, and in the case with the recirculation passage, local to the region affected by flow injection and where geometry optimization is to be carried out. For the rotor case in this paper, a zonal LES calculation, where LES is used in the fluid domain at high span and URANS ev- 


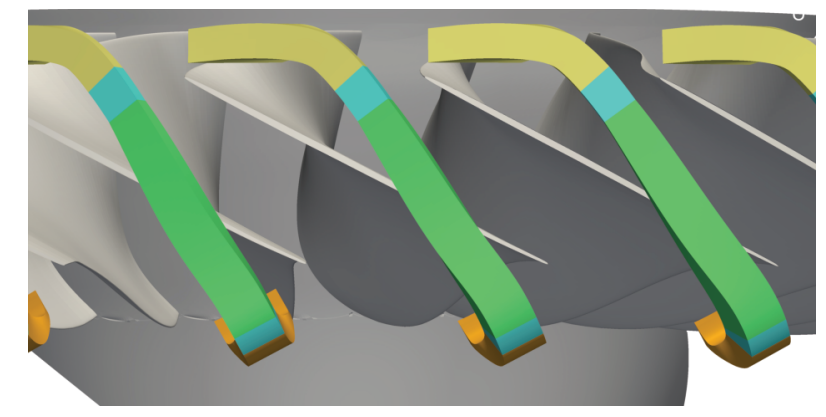

Figure 10: Plan view of the recirculation passage in the casing of a one-third annulus rotor geometry.

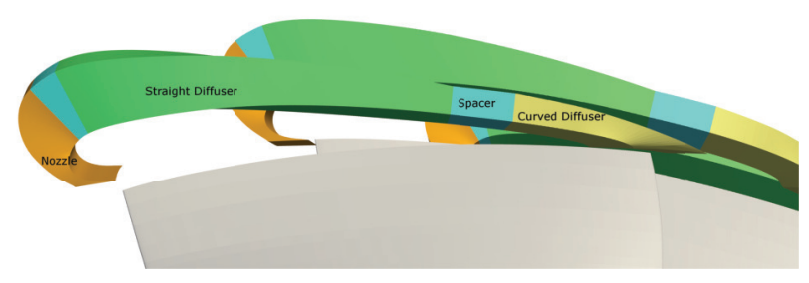

Figure 11: Side view of the recirculation passage showing the diffuser sections.

erywhere else, would capture the complex tip leakage stall inception mechanism at even higher fidelity. This would lead to a more detailed understanding of the stall mechanisms for different distortions. High span zonal LES would also give a higher fidelity calculation of the turbulent mixing of the injected jet from a recirculation passage. This would move practical unsteady CFD closer to the predictive capability of full LES and may facilitate detailed design optimization of the passage itself. 


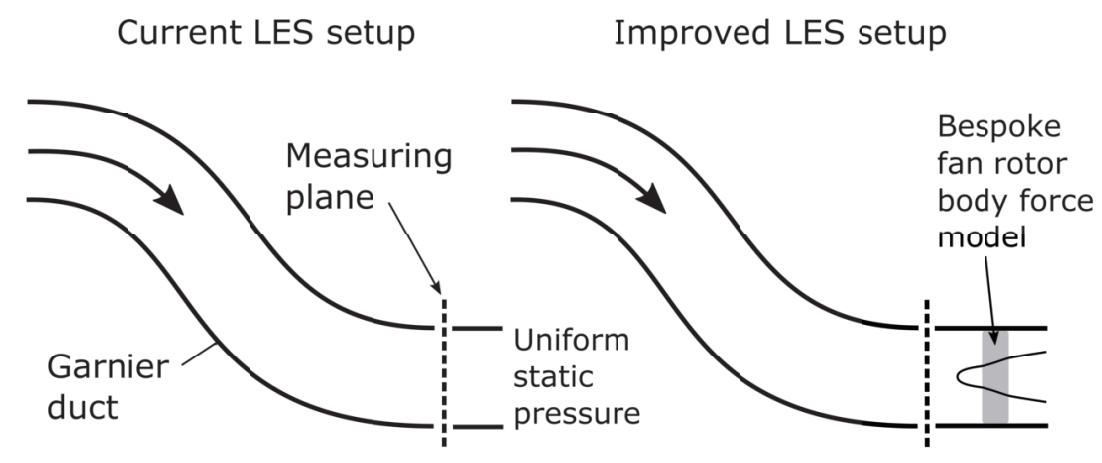

Figure 12: Schematic diagrams of the current and future setups of duct LES.

\section{ACKNOWLEDGEMENT}

The authors would like to thank Rolls-Royce plc for permission to publish and to use inhouse software. Rob Watson is gratefully acknowledged for permission to use LES results, and also Tobias Brandvik, Graham Pullen and John Denton for permission to use Turbostream.

\section{REFERENCES}

[1] P. G. Tucker. Unsteady Computational Fluid Dynamics in Aeronautics. Fluid Mechanics and its Applications, 2014.

[2] J. D. Denton. Some Limitations of Turbomachinery CFD. In Proc. ASME Turbo Expo 2010, June 2010. ASME Paper No. GT2010-22540.

[3] M. J. Shaw, P. Hield, and P. G. Tucker. The Effect of Inlet Guide Vanes on Inlet Flow Distortion Transfer and Transonic Fan Stability. ASME J. Turbomach., 136, Feb 2014.

[4] C. S. Mistry and A. M. Pradeep. Investigations on the Effect of Inflow Distortion on the Performance of a High Aspect Ratio, Low Speed Contra Rotating Fan Stage. In Proc. ASME Turbo Expo 2013, June 2013. ASME Paper No. GT2013-94311.

[5] E. J. Gunn, S. E. Tooze, C. A. Hall, and Y. Colin. An Experimental Study of Loss Sources in a Fan Operating With Continuous Inlet Stagnation Pressure Distortion. ASME J. Turbomach., 135, Sep 2013.

[6] E. J. Gunn and C. A. Hall. Aerodynamics of Boundary Layer Ingesting Fans. In Proc. ASME Turbo Expo 2014, June 2014. ASME Paper No. GT2014-26142.

[7] D. Perovic, C. A. Hall, and E. J. Gunn. Stall Inception In A Boundary Layer Ingesting Fan. In Proc. ASME Turbo Expo 2015, June 2015. ASME Paper No. GT2015-43025.

[8] R. Watson, P. G. Tucker, and K. Menzies. Unsteady Simulations of the Wellborn Diffusing S-Duct. In 54th AIAA Aerospace Sciences Meeting, AIAA Science and Technology Forum and Exposition, Jan 2016.

[9] T. Cao, Hield P., and P. G. Tucker. Hierarchical Immersed Boundary Method with Smeared Geometry. In 54th AIAA Aerospace Sciences Meeting, AIAA Science and Technology Forum and Exposition, Jan 2016. 
[10] J. H. Page, P. Hield, and P. G. Tucker. Inverse Design of 3D Multistage Transonic Fans at Dual Operating Points. ASME J. Turbomach., 136, Apr 2014.

[11] T. Brandvik and G. Pullen. An Accelerated 3D Navier-Stokes Solver for Flows in Turbomachines. ASME J. Turbomach., 133, 2011.

[12] J. H. Page, P. Hield, and P. G. Tucker. Effect of Vortex Ingestion on Transonic Fan Stability. In 54th AIAA Aerospace Sciences Meeting, AIAA Science and Technology Forum and Exposition, Jan 2016.

[13] G. Pullan, A.M. Young, I.J. Day, E.M. Greitzer, and Z.S. Spakovsky. Origins and Structure of Spike-Type Rotating Stall. Journal of Turbomachinery, 137, 2015.

[14] C. Guinet, J. A. Streit, and V. Gümmer. Tip Gap Variation on a Transonic Rotor in the Presence of Tip Blowing. In Proc. ASME Turbo Expo 2014, June 2014. ASME Paper No. GT2014-25042.

[15] C. Guinet, A. Inzenhofer, and V. Gümmer. Influencing Factors of Tip Blowing Interacting with Rotor Tip Flow. In Proc. ASME Turbo Expo 2015, June 2015. ASME Paper No. GT2015-42039.

[16] D.S. Miller. Internal Flow Systems. Miller Innovations, 2 edition, April 2009. 